\title{
Research on Detection Method of Abnormal Behavior of People in Video Surveillance
}

\author{
Bo Zhai \\ College of Computer and Communication Engineering, Beijing Information Technology College, Beijing, \\ China, 100018
}

Keywords: video surveillance; abnormal behavior of people; detection

\begin{abstract}
In view of the abnormal behaviors of all kinds of people, this paper starts with the types and characteristics of abnormal behaviors of people. After fully understanding the characteristics of abnormal behaviors of people, three groups of abnormal behaviors feature extraction methods are selected. The abnormal movement behavior, color and texture features of people in the monitoring are extracted, and on this basis, SVM is used to detect and identify abnormal behavior characteristics of people in video surveillance, in order to provide reference for the detection of abnormal behaviors of people in video surveillance.
\end{abstract}

\section{Introduction}

In recent years, with the improvement of the national economy of our country, the number of large-scale events held throughout the country has become frequent. In this context, various types of mass incidents have begun to occur frequently, such as terrorist attacks, stampede accident, etc. which have many characteristics such as great harm and wide range of influence, which seriously threatens the personal safety of our people. At present, as far as social security work is concerned, how to detect group emergencies in a timely manner and quickly control the incident scene becomes a problem worth considering, and it makes the abnormal identification of the crowd a new means. The problem of abnormal identification of crowds is usually starts with the anomaly feature extraction and anomaly modeling techniques to identify abnormal features, and this step is the strict feature extraction - abnormal identification steps. First, it extracts features of abnormal populations, then compares them with their features to determine their abnormal behavior. In terms of feature extraction, some scholars proposed to first analyze the texture of the human contour region [1], then use the Gaussian kernel function to denoise the texture, and finally reduce the dimension and cluster the features through PCA principal component analysis. It provides the basis for subsequent anomaly classification, in literature [2], based on the extraction of traditional anomaly features, an analysis of crowd escape anomaly based on Bayesian classification model is proposed. In this study, the author describes the optical flow field, and the related class conditional probability density function is constructed by field properties. Finally, the above features are classified by the Bayesian model. The test results show that this step is more suitable for the detection of escaped people. However, experiments have also shown that this method is not suitable for high-density people. The main reason is that in high-density situations, crowd evasive behavior and low or medium density crowded scene characteristics are different.

\section{Types and Characteristics of Abnormal Behaviors of People}

\subsection{Types of abnormal behaviors of people}

Abnormal behavior generally refers to behavior that is contrary to social civilization and group behavior. At present, the crowd's abnormal behavior is mainly divided into two major types, which are abnormal violence behavior and non-violent abnormal behavior. Among them, abnormal behavior of violence refers to abnormal behavior with high exercise capacity, such as trampling, gang fights, etc. This kind of crowd abnormal behavior will seriously threaten people's life and 
property safety once they occur, non-violent abnormal behavior refers to abnormal behavior that indirectly causes losses to people.

\subsection{Characteristics of abnormal behaviors of people}

Under normal circumstances, crowd abnormal behavior generally occurs when the population is highly dense and the exercise pattern is abnormal. Therefore, most of the current crowd abnormal behavior detection methods are based on population density and carry out a series of work such as feature extraction. In a group of activities, when the population density exceeds a certain standard threshold, this part of the crowd exceeding the threshold is likely to have abnormal crowd behavior. It can be seen that how to extract abnormal behavior characteristics of the crowd is the basis for the study of the detection methods of crowd abnormal behavior in video surveillance.

\section{Selection Extraction Methods of Abnormal Behavioral Characteristics and Extraction Process Analysis}

Through the above analysis, we know that the appropriate method for extracting abnormal behaviors of the crowd is the key to determining whether the detection method of crowd abnormal behavior in video surveillance is effective. However, due to the complex background and crowded characteristics in the process of crowd abnormal behavior, it is difficult to extract abnormal characteristics of the crowd. In this regard, this article will start from the abnormal behavior of the crowd, color and texture features, and use three different feature extraction methods to extract the abnormal behavior characteristics of the crowd. Specifically, as follows:

\subsection{Feature extraction of abnormal behaviors of people based on optical flow method}

The optical flow method is a simple and practical image motion expression method. It was studied and proposed in the 1950s, and it is intuitive, clear, and easy to understand. It has been widely used in the detection of moving targets and other important recognition fields to extract motion features [3-6]. In the process of application, the optical flow method will not be affected by the complex background of the movement, so it can directly extract the speed and direction of the abnormal behavior of the crowd, providing a basis for the follow-up crowd abnormal behavior detection. Therefore, this article will select the optical flow method for video surveillance in the crowd abnormal behavior movement feature extraction.

\subsection{RGB-based color feature extraction abnormal behaviors of people}

In the aspect of color feature extraction of crowd abnormal behavior in video surveillance, this article will use RGB color system to extract. The nature of RGB is the dominant color standard in the industry, and it has now become the most widely used color system. In the RGB-based crowd abnormal behavior color feature extraction, the author selected RGB total 6-dimensional data to monitor the color change of the abnormal behavior of the crowd in the video, and as a basis for the occurrence of abnormal behavior to judge.

\subsection{Texture feature extraction based on gabor wavelet transform}

As early as the late 20th century, scholars have studied the texture features of crowds and used them as the main basis for calculating crowd density. Therefore, in the field of image extraction and image analysis, texture feature extraction has always occupied an extremely important position. Gabor wavelet transform texture feature extraction method has good directional selectivity and spatial locality. It can extract the image texture information and texture direction information of the crowd in the crowded and complex environment background, which is perfectly suited for application to the crowd of video surveillance in this process of extracting texture features.

Based on this, this paper will select the above three feature extraction methods to extract the abnormal behavior characteristics of crowds in video surveillance. By integrating the abnormal behavioral movement, color and texture features of the crowd, a 166-dimension combination feature is formed, including 120-dimensional movement features, 6-dimensional color features and 
40-dimensional texture features. In the group of abnormal behavior of the whole population, the proportion of the movement features is the largest, followed by texture features and color features. It can be seen that when abnormal crowd behavior occurs in video surveillance, the abnormal behavior category can be judged by the proportion of the movement characteristics in the abnormal behavior group of the crowd. If the proportion is too large, it means that the abnormal behavior is generally a terrorist attack, gang fight, etc. Color characteristics can be used as an auxiliary feature to determine the type of terrorist attacks, and determine whether explosions, shootings, etc. occur in the process of abnormal behavior, texture features are mainly used to judge the degree of crowding. Although the three features have different functions, the relationship between the three is not independent of each other, but as a whole feature data.

\section{SVM-based Identification of Abnormal Behaviors of People}

After completing the extraction of abnormal behaviors of the crowd in video surveillance, it is also necessary to judge whether the characteristics of each behavior are normal or not. In this regard, this paper will use SVM (Support Vector Machine) to identify the characteristics of crowd abnormal behavior in video surveillance. The specific application steps are [7-10]: First, before the identification, the extracted abnormal behavior of the crowd is divided into normal and abnormal categories, and the corresponding marking work is done. Second, SVM is used to train the behavioral feature sequence to obtain a hyperplane that can be classified as a basis for normal behavior, finally, the behavioral feature sequence of the verification set is used. Each abnormal behavior characteristic is verified to identify abnormal behavior characteristics. The following will analyze the SVM optimal hyperplane calculation process and the abnormal behavior detection in the video surveillance:

\subsection{SVM optimal hyperplane calculation process}

In order to obtain better generalization ability, the SVM support vector machine needs to consider the minimum classification error and the structure of the classification line at the same time. The specific calculation process is as follows:

When the SVM encounters the linear separability problem in the process of finding the optimal hyperplane, suppose there is a hyperplane, $\mathrm{w} \times \mathrm{x}+\mathrm{b}=0, w \in R^{N}, b \in R$, and in this case, the characteristics $\left(x_{1}, y_{1}\right), \ldots,\left(x_{i}, y_{i}\right), \ldots,\left(x_{I}, y_{I}\right), x \in R^{N}, y \in\{+1,-1\}$ can be divided into two categories, which are expressed as:

$$
y_{i}[(w \times x)+b] \geq 1, i=1, \ldots, I
$$

After corresponding mathematical calculations, it was found that there is a spatial separation

between the two types of features $\frac{2}{\|w\|}$. There are multiple hyperplanes of this type, and the optimal hyperplane is the hyperplane with the largest spatial separation between the two types of features. In other words, if you want to find the optimal hyperplane, you also need to start with two kinds of feature space interval values, and find the maximum value of the two types of feature space intervals by the following formula:

$$
\left\{\begin{array}{c}
\min \left\{\phi(w)=\frac{1}{2}\|w\|^{2}\right\} \\
y_{i}[(w \times x)+b] \geq 1, i=1, \ldots, I
\end{array}\right.
$$

Using Lagrange Long-Day Functions to Solve the Optimal Hyperplane:

$$
L(w, b, a)=\frac{1}{2}\|w\|^{2}-\sum_{i=1}^{I} a_{i}\left\{\left[\left(x_{i} \times w\right)+b\right] y_{i}-1\right\}
$$


When SVM encounters a nonlinear and inseparable problem in the process of finding the optimal hyperplane, SVM will use the nonlinear transformation method to transform the nonlinear inseparable problem into a linear problem existing in a high latitude space. In the process of transforming non-linear and inseparable problems, SVM can simplify the non-linear latitude space by simply computing the high-latitude space inner product.

\subsection{SVM detection process of abnormal behaviors of people}

The detection and alarm process of crowd abnormal behavior in video surveillance by SVM is mainly divided into the following four steps:

First, using the optical flow method, RGB and Gabor wavelet transform to extract the characteristics of abnormal behavior in the monitoring, and combining the characteristics of each behavior into 166-dimensional feature data. Second, use the SVM to classify the feature dataset, mark the characteristics of abnormal behaviors of various groups of people, and use the label to indicate aggregation or stamping behavior, labe 2 indicates terrorist attack behavior, labe 3 indicates gang fight, and labe4 indicates normal behavior. After the SVM has trained the abnormal behavior of the crowd, if the labe $1 / 2 / 3$ flag appears, it means that the behavior is abnormal. The SVM immediately initiates an alarm to the public security department and continues to detect the extracted behavior after the alarm is initiated. In order to prevent further expansion of abnormal behavior of the crowd. Thirdly, for the sake of detection efficiency and accuracy, this article will take the event with the highest frequency in every 10 frames of images as the output of detection behavior. The specific formula is:

$$
\text { label }=\max \left(\text { num }_{\text {label } \_} \text {all }\right)
$$

In formula (4), Label represents the final output of every 10 frames of image.

Fourth, in order to avoid the use of efficiency in the detection aspect, in order to avoid constant abnormal alarm during the detection process. The author believes that the corresponding matlab statement can be added during the SVM detection process so that the final detection result is always a new alarm.

\section{Test Verification}

In order to verify the scientific and rationality of the detection method of crowd abnormal behaviors in video surveillance proposed in this paper, the above schemes were verified by combining relevant experimental platforms to verify the feasibility and accuracy of the above schemes.

\subsection{Subjects}

Before the experiment began, the author collected some targeted videos of abnormal behavior of the crowd through the Internet platform. They were terrorist attacks, people trampled and gang fight, and formed an abnormal crowd behavior video set as the subject of this experiment. The experimental content is mainly divided into two parts, namely the training sample part and the test sample part. The entire experiment process was conducted in strict accordance with the crowd abnormal behavior detection method in video surveillance proposed in this paper.

\subsection{Experimental results}

This experiment contains a total of three types of training data, namely: people stampede, terrorist attacks and gang fight. The abnormal behavior of the crowd extracted from experimental videos is 166-dimensional. The specific experimental results are shown in Table 1. 
Table 1 Experimental results

\begin{tabular}{ccccc}
\hline Behavior & $\begin{array}{c}\text { Detection } \\
\text { times }\end{array}$ & $\begin{array}{c}\text { Number of false } \\
\text { positives }\end{array}$ & $\begin{array}{c}\text { False } \\
\text { alarm rate }\end{array}$ & Correct rate \\
\hline People Stampede & 80 & 22 & $27.5 \%$ & $72.5 \%$ \\
Terrorist attacks & 86 & 28 & $32.5 \%$ & $68.5 \%$ \\
Gang fight & 79 & 27 & $34.2 \%$ & 65.9 \\
\hline
\end{tabular}

According to the experimental results, it can be seen that in the process of application of crowd abnormal behavior detection in video surveillance proposed in this paper, the detection accuracy rate is much higher than the false alarm rate. This shows that the above studies are reasonable and scientific.

\section{Conclusion}

To sum up, a set of sound detection methods for crowds in video surveillance is the key to stabilizing public order. In the course of this research, the author proposes to extract the characteristics of abnormal behavior in video surveillance by using optical flow method, RGB and Gabor wavelet transform, and then use SVM to train feature sequences to detect crowd abnormalities in video surveillance. Behavioural and promptly initiate an alert to the security department. The empirical part of this paper proves that this method is realistic and can support the smooth progress of social security work.

\section{Acknowledgement}

General project of science and technology program of Beijing Education Committee XY-YW$02-201602$

\section{References}

[1] Xinyu W, Liang GY, Lee KK, Xu YS. Crowd Density Estimation Using Texture Analysis and Learning. IEEE International Conference on Robotics and Biomimetics. Kunming. Institute of Electrical and Electronics Engineers. 2006. 214-219

[2] $21 \mathrm{Wu}$ S, Wong HS, Yu Z. A Bayesian Model for Crowd Escape Behavior Detection. IEEE Trans. on Circuits and Systems for Video Technology, 2014, 24(1): 85-98.

[3] Shang Liu, Linfang Dong. Anomaly Detection Algorithm for Crowd Movement Direction [J]. Computer Science, 2013, 40(S2): 337-340.

[4] Pengyue Zhang, Liang Zhang. Analysis of Crowd State Combined with Optical Flow Method and Information Entropy [J]. Signal Processing, 2015, 31(09): 1101-1105.

[5] Zheng Shen, Wei Wu. Detection of Abnormalities in Bus Crowd Based on Video Images [J]. Journal of Nanjing University of Science and Technology, 2017, 41(01): 65-73+79.

[6] Ming Yu, Tuantuan Guo. Crowd Anomaly Detection Based on Gaussian Mixture Model [J]. Software Guide, 2017, 16(11): 114-120+2.

[7] Quanzhu Yao, Jie Cai. LS-SVM Feature Selection and Parameter Optimization Algorithm Based on PSO[J]. Computer Engineering and Applications, 2010, 46(01): 134-136+229.

[8] Wengang Ma, Xiaopeng Wang, Zuopeng Wu. PSO-SVM Eigenvector Fall Detection Method Based on Human Pose[J]. Journal of Transduction Technology, 2017, 30(10): 1504-1511.

[9] Weidong Jiao, Shusen Lin. Overall Improved Fault Diagnosis Method Based on Support Vector Machine[J]. Journal of Instrumentation, 2015, 36(08): 1861-1870. 